\title{
Adaptive BDDC in Three Dimensions
}

\author{
Jan Mandel ${ }^{\mathrm{a}}$, Bedřich Sousedík ${ }^{\mathrm{a}, \mathrm{b}}$, Jakub Š́stek ${ }^{\mathrm{c}, \mathrm{d}, *}$ \\ ${ }^{a}$ Department of Mathematical and Statistical Sciences, University of Colorado Denver, \\ Denver, CO 80217-3364, USA. \\ ${ }^{b}$ Institute of Thermomechanics, Academy of Sciences of the Czech Republic, \\ Dolejškova 1402/5, CZ - 18200 Prague 8, Czech Republic. \\ ${ }^{c}$ Institute of Mathematics, Academy of Sciences of the Czech Republic, \\ Žitná 25, CZ - 11567 Prague 1, Czech Republic. \\ ${ }^{d}$ Department of Mathematics, Faculty of Mechanical Engineering, \\ Czech Technical University in Prague, \\ Karlovo náměstí 13, CZ - 12135 Prague 2, Czech Republic.
}

\begin{abstract}
The adaptive BDDC method is extended to the selection of face constraints in three dimensions. A new implementation of the BDDC method is presented based on a global formulation without an explicit coarse problem, with massive parallelism provided by a multifrontal solver. Constraints are implemented by a projection and sparsity of the projected operator is preserved by a generalized change of variables. The effectiveness of the method is illustrated on several engineering problems.
\end{abstract}

Keywords: parallel algorithms, domain decomposition, iterative substructuring, BDDC, adaptive constraints

\section{Introduction}

The Balancing Domain Decomposition by Constraints (BDDC) was developed by Dohrmann [6] as a primal alternative to the Finite Element Tearing and Interconnecting - Dual, Primal (FETI-DP) by Farhat et al. [7]. Both methods use constraints to impose equality of new "coarse" variables on substructure interfaces, such as values at substructure corners or weighted averages over edges and faces. Primal variants of the FETI-DP were also independently proposed by Cros [4] and by Fragakis and Papadrakakis [10]. It has been shown in [27, 37] that these methods are in fact the same as BDDC. Polylogarithmic condition number bounds for FETI-DP were first proved in [29] and generalized to the case of coefficient jumps between substructures in [14]. The same bounds were obtained for BDDC in [23, 24]. A proof that the eigenvalues of the preconditioned operators of both methods are actually the same except for the eigenvalues equal to one was given in [24] and then simplified

*Corresponding author; tel.: +420 222090 710; fax: +420 222211638.

Email addresses: jan.mandel@ucdenver.edu (Jan Mandel), bedrich.sousedik@ucdenver.edu (Bedřich Sousedík), sistek@math.cas.cz (Jakub Š́stek) 
in [2, 20, 27]. FETI-DP, and, equivalently, BDDC are quite robust. It can be proved that the condition number remains bounded even for large classes of subdomains with rough interfaces in 2D [12, 39] as well as in many cases of strong discontinuities of coefficients, including some configurations when the discontinuities cross substructure boundaries [31, 32]. However, the condition number deteriorates in many situations of practical importance and a better selection of constraints is desirable. Enriching the coarse space so that the iterations run in a subspace devoid of "difficult" modes has been a successful trick in iterative substructuring methods used, e.g., in the development of BDD and FETI for plates from the base BDD and FETI methods [8, 18, 19, 30]. Methods that build a coarse space adaptively from local eigenvalue calculations were also devised in other (though related) contexts [3, 9, 21, 22, 33. Adaptive enrichment for BDDC and FETI-DP was proposed in [25, 26], with the added coarse functions built from eigenproblems based on adjacent pairs of substructures in 2D. The adaptive method, however, was formulated in terms of FETI-DP operators, and it was quite complicated.

Here, we develop the adaptive algorithm directly in terms of BDDC operators, resulting in a much simpler formulation and implementation. Of course, the algorithm still allows a translation into the language of the FETI-DP. We then extend the construction from [25, 26] to 3D. We find that the heuristic eigenvalue-based estimates still work reasonably well and that our adaptive approach can result in the concentration of computational work in a small troublesome part of the problem, which leads to a good convergence behaviour at a small added cost.

We also develop a new implementation framework that operates on global matrices, builds no explicit coarse problem, and gets much of its parallelism through the direct solver used for solution of an auxiliary decoupled system. To preserve sparsity, we use a variant of the change of variables from [20], extended to an arbitrary number of constraints. Our current parallel implementation is built on top of the multifrontal massively parallel sparse direct solver MUMPS [1, motivated also by an earlier implementation of the BDDC preconditioner based on the frontal solver [34].

The rest of the paper is organised as follows. In Section 2, we establish the notation and review the BDDC algorithm in a form suitable for our purposes. In Section 3, we describe the adaptive method. Section 4 then describes the implementation on top of a massively parallel direct solver. Section 5 presents the generalized change of variables to preserve sparsity. Section 6 describes some further details of the implementation. Numerical results are presented in Section 7. Section 8 contains the summary and concluding remarks.

Some of results in this paper were presented in the thesis [36].

\section{Notation, substructuring, and BDDC}

To establish notation, we first briefly review standard substructuring concepts and state the BDDC method in a form suitable for our purposes. The setting and notation here is compatible with [28], with some additions. See, e.g., [35, 38] for more details about iterative substructuring and [16, 23, 28, 35, 38] for BDDC. 
Consider an elliptic boundary value problem defined on a bounded domain $\Omega \subset \mathbb{R}^{3}$ and discretized by conforming finite elements. The domain $\Omega$ is decomposed into $N$ nonoverlapping subdomains $\Omega_{i}, i=1, \ldots N$, also called substructures, so that each substructure $\Omega_{i}$ is a union of finite elements. Each node is associated with one degree of freedom in the scalar case, and with 3 displacement degrees of freedom in the case of linear elasticity. The nodes contained in more than one substructure are called the interface, denoted by $\Gamma$, and $\Gamma_{i}=\Gamma \cap \Omega_{i}$ is the interface of substructure $\Omega_{i}$. The interface $\Gamma$ may also be classified as the union of three different types of nonoverlapping sets: faces, edges, and corners. We will adopt here the following simple definition. A face contains all nodes shared solely by one pair of subdomains, an edge contains all nodes shared by same set of more than two subdomains, and a corner is a degenerate edge with only one node; for a more general definition see e.g. [13. Edges and faces are also called globs.

We identify finite element functions with the vectors of their coefficients in the standard finite element basis. These coefficients are also called variables or degrees of freedom. We also identify linear operators with their matrices, in bases that will be clear from the context.

The space of all (vectors of the degrees of freedom of) finite element functions on subdomain $\Omega_{i}$ is denoted by $W_{i}$, and let

$$
W=W_{1} \times \cdots \times W_{N} .
$$

The space $W$ is equipped with the standard $\mathbb{R}^{n}$ basis and the Euclidean inner product $\langle w, v\rangle=w^{\mathrm{T}} v$. For a symmetric positive semidefinite matrix $M,\langle u, v\rangle_{M}=\langle M u, v\rangle$, and $\|u\|_{M}=\langle M u, u\rangle^{1 / 2}$.

Let $A_{i}: W_{i} \rightarrow W_{i}$ be the local substructure stiffness matrix, obtained by the subassembly of element matrices only in substructure $\Omega_{i}$. The matrices $A_{i}$ are symmetric positive semidefinite for an elliptic problem. We can write vectors and matrices in the block form

$$
w=\left[\begin{array}{c}
w_{1} \\
\vdots \\
w_{N}
\end{array}\right], \quad w \in W, \quad A=\left[\begin{array}{ccc}
A_{1} & & \\
& \ddots & \\
& & A_{N}
\end{array}\right]: W \rightarrow W .
$$

Now let $U \subset W$ be the space of all functions from $W$ that are continuous across substructure interfaces. We are interested in solving the problem

$$
u \in U: \quad\langle A u, v\rangle=\langle f, v\rangle, \quad \forall v \in U
$$

where $f \in W$ is a given right-hand side. Vectors from $U$ are called vectors of global degrees of freedom, while vectors from $W_{i}$ are called local. The space $U$ is equipped with the basis of 0-1 vectors with one basis vector for each global degree of freedom. The basis vectors have 1s in the places where the global degree of freedom coincides with a local one. The matrix

$$
R: U \rightarrow W
$$

formed from these basis vectors as columns, is the familiar global-to-local mapping that restricts the global vectors of degrees of freedom to local degrees of freedom on each $\Omega_{i}$. 
Thus, $R^{\mathrm{T}} A R$ is the global stiffness matrix, and (3) is equivalent to the assembled system

$$
R^{\mathrm{T}} A R v=R^{\mathrm{T}} f .
$$

The matrix $R$ is also the matrix of the canonical embedding $U \subset W$ in the given bases.

Denote by $U_{I} \subset W$ the space of all (vectors of) finite element functions with nonzero values only in the interiors of substructures $\Omega_{i}$. Then $U_{I} \subset U$, and the space $W$ is decomposed as the $A$-orthogonal direct sum

$$
W=U_{I} \oplus W_{H}, \quad U_{I} \perp_{A} W_{H},
$$

where the functions from $W_{H}$ are called discrete harmonic. Such functions are fully determined by values of degrees of freedom at the interface, and they have minimal energy on every subdomain. Therefore, in a computer implementation, only interface values of discrete harmonic functions need to be stored.

The $A$-orthogonal projection onto $U_{I}$ is denoted by

$$
P: W \rightarrow U_{I} .
$$

For $w \in W,(I-P) w$ is the discrete harmonic extension from the values of $w$ on the substructure boundaries. The evaluation of $P w$ consists of the solution of $N$ independent Dirichlet problems, one in each substructure.

The space of all discrete harmonic functions from $W$ that are continuous at interface is denoted by $\widehat{W}$. We have

$$
\widehat{W}=W_{H} \cap U=(I-P) U,
$$

and the $A$-orthogonal decomposition

$$
U=U_{I} \oplus \widehat{W}, \quad U_{I} \perp_{A} \widehat{W} .
$$

The solution $v \in U$ of problem (5) is split as

$$
R v=u+w, \quad u \in U_{I}, w \in \widehat{W} .
$$

Solving for the interior component $u \in U_{I}$ decomposes into $N$ independent Dirichlet problems. We are interested in finding the discrete harmonic component $w \in \widehat{W}$, which is the solution of the reduced problem

$$
w \in \widehat{W}: \quad\langle A w, z\rangle=\langle f, z\rangle, \quad \forall z \in \widehat{W} .
$$

We further need an averaging operator

$$
E: W \rightarrow U \text {. }
$$

The operator $E$ replaces the variables on the interface by their averages (arithmetic or weighted) from all adjacent subdomains, and it preserves variables in the interiors of substructures. The operator $E$ is a projection from $W$ onto $U$. Then the operator

$$
(I-P) E: W \rightarrow \widehat{W}
$$


is a projection from $W$ onto $\widehat{W}$. Its evaluation consists of averaging between the substructures, followed by the discrete harmonic extension from the substructure boundaries. Also, note that

$$
(I-(I-P) E) w=(I-P)(I-E) w, \quad \forall w \in W_{H}
$$

since $P w=0$ if $w \in W_{H}$.

Proper weights (e.g. proportional to the substructure stiffness) in the averaging given by $E$ are important for the performance of BDDC (as well as other iterative substructuring methods) independent of different stiffness of substructures [13, 24].

The BDDC preconditioner is characterized by a selection of coarse degrees of freedom, such as values at corners and averages over edges or faces. The action of the BDDC preconditioner is then defined in the space given by the requirement that the coarse degrees of freedom on adjacent substructures coincide, which is enforced in the algorithms by constraints. So, the design of the BDDC preconditioner is characterized by a selection of an intermediate space $\widetilde{W}$ satisfying these constraints,

$$
\widehat{W} \subset \widetilde{W} \subset W_{H}
$$

The BDDC then consists of preconditioned conjugate gradients (PCG) applied to the problem (11) with the preconditioner

$$
M_{B D D C}: r \mapsto u=(I-P) E w, \quad w \in \widetilde{W}: \quad\langle A w, z\rangle=\langle r,(I-P) E z\rangle, \quad \forall z \in \widetilde{W},
$$

where $r$ is the residual in the PCG method. The following condition number bound for BDDC will play an essential role in our design of the adaptive method.

Theorem 1 ([24]). The eigenvalues of the preconditioned operator of the BDDC method satisfy $1 \leq \lambda \leq \omega_{B D D C}$, where

$$
\omega_{B D D C}=\sup _{w \in \widetilde{W}} \frac{\|(I-(I-P) E) w\|_{A}^{2}}{\|w\|_{A}^{2}} .
$$

The BDDC enforces the equality of corner coarse degrees of freedom directly by using the space $W^{c}$, consisting of all functions where the local degrees of freedom on the substructure corners coincide. Then

$$
U \subset W^{c} \subset W
$$

Just like $U$, space $W^{c}$ is equipped with a basis consisting of 0 -1vectors. The basis vector corresponding to a corner degree of freedom has 1s in the places where the global degree of freedom coincides with the corresponding substructure degree of freedom. The global-to-local matrix

$$
R^{c}: W^{c} \rightarrow W
$$

formed from these basis vectors as columns, is the matrix of the canonical embedding $W^{c} \subset W$, and

$$
A^{c}=R^{c \mathrm{~T}} A R^{c}
$$


is the stiffness matrix assembled at the subdomain corners only (Figure 1).

We require that there are sufficiently many corner constraints, which leads to the following assumption.

Assumption 1. The matrix $A$ is positive definite on $W^{c}$.

Denote by $\widetilde{W^{c}}$ the space all of discrete harmonic functions in $W^{c}$,

$$
\widetilde{W}^{c}=W^{c} \cap W_{H} \text {. }
$$

Then

$$
\widehat{W} \subset \widetilde{W} \subset \widetilde{W^{c}} \subset W_{H},
$$

and we construct the space $\widetilde{W}$ by enforcing the remaining constraints weakly by a matrix $D$,

$$
\widetilde{W}=\left\{w \in \widetilde{W^{c}}: D w=0\right\} .
$$

Each row of $D$ defines one constraint. We require that the constraints are satisfied by all functions that are continuous across the interfaces,

$$
D w=0, \quad \forall w \in U .
$$

Note that 24 implies that $\widehat{W} \subset \widetilde{W}$, and that the constraints $D w=0$ involve boundary variables only. The adaptive algorithm will construct such matrix $D$.

Lemma 1. The BDDC preconditioner (16) satisfies

$$
M_{B D D C}: r \mapsto u=(I-P) E R^{c} w_{c} .
$$

For some $\lambda$, it holds

$$
\begin{array}{llc}
A^{c} w_{c}+D^{c \mathrm{~T}} \lambda & = & R^{c \mathrm{~T}} E^{\mathrm{T}}(I-P)^{\mathrm{T}} r \\
D^{c} w_{c} & = & 0
\end{array}
$$

where

$$
D^{c}=D R^{c}
$$

differs from $D$ only by omitting some zero columns corresponding to corners.

Proof. The saddle point problem (26) is equivalent to the constrained minimization

$$
\frac{1}{2}\langle A w, w\rangle-\langle r,(I-P) E w\rangle \rightarrow \min \quad \text { subject to } w \in W^{c}, D w=0,
$$

with $w=R^{c} w_{c}$. Let $w$ be a solution of (28) and $z \in U_{I}$. Since $w$ is optimal with respect to variation $z$, and $(I-P) z=0$, we have $\langle A w, z\rangle=0$. Thus, $w$ is discrete harmonic. It follows that 28 is equivalent to

$$
\frac{1}{2}\langle A w, w\rangle-\langle r,(I-P) E w\rangle \rightarrow \min \quad \text { subject to } w \in \widetilde{W}^{c}, D w=0,
$$

which, by (23), is the same as 16$)$.

Finally, matrix $D R^{c}$ differs from $D$ only by omitting zero columns because the constraints $D w=0$ do not involve corners. 
Remark 1. In practice, the computation of $(I-P)^{\mathrm{T}} r$ can be omitted, because $r=$ Ae, where the error $e$ is discrete harmonic, and then

$$
\left\langle P^{\mathrm{T}} r, z\right\rangle=\left\langle P^{\mathrm{T}} A e, z\right\rangle=\langle A e, P z\rangle=\langle e, z\rangle_{A}=0, \quad \forall z \in U_{I},
$$

thus $P^{\mathrm{T}} r=0$, that is, $r=0$ in the interiors. The condition that the error $e$ is discrete harmonic is preserved in the iteration by induction, and the initial error can be made discrete harmonic by a suitable choice of initial approximation for the reduced problem (e.g., zero).

\section{Adaptive selection of constraints}

We first briefly review the principle of the adaptive method from [26], in a form suitable for our purposes. The condition number bound $\omega_{B D D C}$ from Theorem 1 equals to the maximum eigenvalue $\lambda_{1}$ of the associated generalized eigenvalue problem

$$
w \in \widetilde{W}: \quad\langle(I-(I-P) E) w,(I-(I-P) E) z\rangle_{A}=\lambda\langle w, z\rangle_{A}, \quad \forall z \in \widetilde{W} .
$$

The following statement is well known from linear algebra, e.g. [5, Theorem 5.2].

Lemma 2 (Courant-Fisher-Weyl minimax principle). Let $c(\cdot, \cdot)$ be symmetric positive semidefinite bilinear form on vector space $V$ of dimension $n$ and $b(\cdot, \cdot)$ symmetric positive definite bilinear form on $V$. Then the generalized eigenvalue problem

$$
w \in V: c(w, u)=\lambda b(w, u), \quad \forall u \in V
$$

has $n$ linearly independent eigenvectors $w_{k}$ and the corresponding eigenvalues are real and nonnegative and the eigenvectors are stationary points of the Rayleigh quotient $c(w, w) / b(w, w)$, with the stationary values equal to $\lambda_{i}$. Order $\lambda_{1} \geq \lambda_{2} \geq \ldots \geq \lambda_{n} \geq 0$. Then, for any subspace $V_{k} \subset V$ of dimension $n-k$,

$$
\max _{w \in V_{k}, w \neq 0} \frac{c(w, w)}{b(w, w)} \geq \lambda_{k+1}
$$

with equality if

$$
V_{k}=\left\{w \in V: c\left(w_{\ell}, w\right)=0, \quad \forall \ell=1, \ldots, k\right\} .
$$

Since the bilinear form on the left-hand side of (31) is symmetric positive semidefinite and the bilinear form on the right-hand side is symmetric positive definite, Lemma 2 applies and leads to the following corollary.

Corollary 1. The generalized eigenvalue problem (31) has eigenvalues $\lambda_{1} \geq \lambda_{2} \geq \ldots \geq$ $\lambda_{n} \geq 0$. Denote the corresponding eigenvectors $w_{\ell}$. Then, for any $k=1, \ldots, n-1$, and any linear functionals $L_{\ell}$ on $W, \ell=1, \ldots, k$,

$$
\max \left\{\frac{\|(I-(I-P) E) w\|_{A}^{2}}{\|w\|_{A}^{2}}: w \in \widetilde{W}, L_{\ell}(w)=0, \quad \forall \ell=1, \ldots, k\right\} \geq \lambda_{k+1},
$$

with equality if

$$
L_{\ell}(w)=\left\langle(I-(I-P) E) w_{\ell},(I-(I-P) E) w\right\rangle_{A} .
$$


The next lemma shows that the added constraints $L_{\ell}(w)=0$ satisfy the compatibility condition (24).

Lemma 3. The constraints $L_{\ell}(w)=0$, with $L_{\ell}$ given by (35), are satisfied for any $w \in U$.

Proof. From (14), $(I-(I-P) E)=(I-P)(I-E)$. For any $w \in U,(I-E) w=0$, because $E$ is a projection on $U$.

It follows that the optimal decrease of the condition number bound $(17)$ can be achieved by adding the rows $d_{\ell}^{\mathrm{T}}$ defined by $d_{\ell}^{\mathrm{T}} w=L_{\ell}(w)$ to the constraint matrix $D$ in the definition of $\widetilde{W}(23)$.

However, solving the global eigenvalue problem (31) is expensive, and the vectors $d_{\ell}$ are not of the form suitable for substructuring, i.e., each $d_{\ell}$ with nonzeros at one glob only. For these reasons, we replace (31) by a collection of local problems, each defined by considering only two adjacent subdomains $\Omega_{i}$ and $\Omega_{j}$ at a time. Here, subdomains are considered adjacent if they share an edge in 2D, or a face in 3D (Figure 2). All quantities associated with such pair will be denoted by the subscript $i j$. Using also (14), the generalized eigenvalue problem (31) becomes

$w_{i j} \in \widetilde{W}_{i j}: \quad\left\langle\left(I-P_{i j}\right)\left(I-E_{i j}\right) w_{i j},\left(I-P_{i j}\right)\left(I-E_{i j}\right) z_{i j}\right\rangle_{A_{i j}}=\lambda\left\langle w_{i j}, z_{i j}\right\rangle_{A_{i j}}, \quad \forall z_{i j} \in \widetilde{W}_{i j}$.

Assumption 2. The corner constraints are already sufficient to prevent relative rigid body motions of any pair of adjacent substructures, so

$$
\forall w_{i j} \in \widetilde{W}_{i j}: A_{i j} w_{i j}=0 \Rightarrow\left(I-E_{i j}\right) w_{i j}=0,
$$

i.e., the corner degrees of freedom are sufficient to constrain the rigid body modes of the two substructures into a single set of rigid body modes, which are continuous across the interface $\Gamma_{i j}$.

The maximal eigenvalue $\omega_{i j}$ of (36) is finite due to Assumption 2, and we define the heuristic condition number indicator

$$
\widetilde{\omega}=\max \left\{\omega_{i j}: \Omega_{i} \text { and } \Omega_{j} \text { are adjacent }\right\} .
$$

Considering two adjacent subdomains $\Omega_{i}$ and $\Omega_{j}$ only, we get the added constraints $L_{\ell}(w)=0$ from $(35)$ as

$$
\left\langle\left(I-P_{i j}\right)\left(I-E_{i j}\right) w_{i j, \ell},\left(I-P_{i j}\right)\left(I-E_{i j}\right) w\right\rangle_{A_{i j}}=0, \quad \forall \ell=1, \ldots, k_{i j},
$$

where $w_{i j, \ell}$ are the eigenvectors corresponding to the $k_{i j}$ largest eigenvalues from (36).

Algorithm 1 (Adaptive BDDC). Find the smallest $k_{i j}$ for each pair of adjacent substructures $\Omega_{i}$ and $\Omega_{j}$ to guarantee that $\lambda_{i j, k_{i j}+1} \leq \tau$, where $\tau$ is a given tolerance, and add the constraints (39) to the definition of $\widetilde{W}$. 
The adaptive BDDC method assures that the condition number indicator $\widetilde{\omega} \leq \tau$ with the minimum number of added constraints. It was presented in [26] starting from corner constraints only, formulated in terms of FETI-DP, and the result translated to BDDC. We extend the method to the case a general space $\widetilde{W}$ and give a much simpler implementation in BDDC directly.

To formulate a numerical algorithm, we need to write the generalized eigenvalue problem (36) and the added constraints (39) in terms of matrices and vectors. Consider the space $\widetilde{W}_{i j}$ given by the corner constraints and an initial constraint matrix $D_{i j}^{c}$ devised from an initial global matrix $D^{c}$. Recall that $W_{i j}^{c}$ is the space of functions from $W_{i j}$ that are continuous at corners, $R_{i j}^{c}: W_{i j}^{c} \rightarrow W_{i j}$ is the identity embedding, $A_{i j}^{c}=R_{i j}^{c \mathrm{~T}} A_{i j} R_{i j}^{c}$ is the matrix assembled at the corners, and $D_{i j}^{c}=D_{i j} R_{i j}^{c}$. Let

$$
\Pi_{i j}=I-D_{i j}^{c \mathrm{~T}}\left(D_{i j}^{c} D_{i j}^{c \mathrm{~T}}\right)^{-1} D_{i j}^{c}
$$

be the orthogonal projection onto null $D_{i j}^{c}, \Pi_{i j}: \widetilde{W}_{i j}^{c} \rightarrow \widetilde{W}_{i j}$. The initial constraint matrix $D_{i j}^{c}$ can be empty; then $\Pi_{i j}=I$. The generalized eigenvalue problem (31) now becomes

$$
\Pi_{i j}\left(I-E_{i j}\right)^{\mathrm{T}} S_{i j}^{c}\left(I-E_{i j}\right) \Pi_{i j} w_{i j}=\lambda_{i j} \Pi_{i j} S_{i j}^{c} \Pi_{i j} w_{i j},
$$

where

$$
S_{i j}^{c}=\left(I-P_{i j}\right)^{\mathrm{T}} A_{i j}^{c}\left(I-P_{i j}\right)
$$

Since

$$
\operatorname{null} \Pi_{i j} S_{i j}^{c} \Pi_{i j} \subset \operatorname{null} \Pi_{i j}\left(I-E_{i j}\right)^{\mathrm{T}} S_{i j}^{c}\left(I-E_{i j}\right) \Pi_{i j},
$$

the eigenvalue problem (41) reduces in the factorspace modulo null $\Pi_{i j} S_{i j}^{c} \Pi_{i j}$ to a problem with the operator on the right-hand side positive definite. In some computations, we have used the subspace iteration method LOBPCG [15] to find the dominant eigenvalues and their eigenvectors. The LOBPCG iterations then run in the factorspace. To use standard eigenvalue solvers, (41) may be converted to a matrix eigenvalue problem by penalizing the components in null $D_{i j}^{c}$ and rigid body modes, as already described in [26].

It follows from the matrix form of the eigenvalue problem (43), that the constraints to be added are

$$
L_{i j, \ell}\left(w_{i j}\right)=w_{i j, \ell}^{\mathrm{T}} \Pi_{i j}\left(I-E_{i j}\right)^{\mathrm{T}} S_{i j}^{c}\left(I-E_{i j}\right) \Pi_{i j} w_{i j}=0,
$$

where $w_{i j}$ is the restriction of $w$ to the pair of subdomains. That is, we wish to add to the constraint matrix $D$ the rows

$$
d_{i j, \ell}=w_{i j, \ell}^{\mathrm{T}} \Pi_{i j}\left(I-E_{i j}\right)^{\mathrm{T}} S_{i j}^{c}\left(I-E_{i j}\right) \Pi_{i j} .
$$

These rows are extended to the size of global matrix $D$ simply by zeros.

Proposition 1. The vectors $d_{i j, \ell}$, constructed for a domain consisting of only two substructures $\Omega_{i}$ and $\Omega_{j}$, have matching entries on the interface between the two substructures, with opposite signs. 
Proof. Consider the vector $w \in W$ that has two entries equal to 1, corresponding to a degree of freedom on the interface, and all other entries equal to 0. Using the definition of $d_{i j, \ell}$ (45) and Lemma 3, we get $d_{i j, \ell} w_{i j}=L_{i j, \ell}\left(w_{i j}\right)=0$. The proof is concluded by taking $w_{i j}$ of this form for arbitrary degree of freedom, all of which satisfy relation (44).

In $2 \mathrm{D}$, one can simply add rows (45) to the constraint matrix $D$, which is equivalent to the method from [26]. In 3D, unfortunately, such rows would generally have nonzero entries over all of the interface of $\Omega_{i}$ and $\Omega_{j}$, including the edges (where $\Omega_{i}$ and $\Omega_{j}$ intersect other substructures). Consequently, these rows would couple several globs together, and the matrix $D^{c} D^{c \text { T }}$ would be in general no longer block diagonal with one block per glob. To preserve the block diagonal structure, we have to split each $d_{i j, \ell}$ into one row that contains the nonzero entries of the face, and one row for each edge that contains the nonzero entries of that edge. From Proposition 1, it follows that these split constraints satisfy the compatibility condition (24), and thus the space $\widetilde{W}$ is well defined.

Remark 2. In the computations reported in Section 7, we drop the adaptively generated edge constraints in 3D. Then it is no longer guaranteed that the condition number indicator $\widetilde{\omega} \leq \tau$. However, the method is still observed to perform well.

\section{Parallel framework with global matrices and on top of multifrontal solver}

The main purpose of BDDC, just like any other iterative substructuring method, is to split the problem into subproblems, which are solved independently on separate nodes in a multiprocessor system. Therefore, the usual implementation results in independent local problems on the spaces $W_{i}$ and a small coarse problem [6, 23]. Parallel implementation then requires a fair amount of custom coding. To reduce the amount of new code, a BDDC implementation that uses specially crafted calls to a frontal solver to compute almost all quantities on the substructures was developed [34. However, the frontal solver implementation needs to construct a coarse problem, and the programmer needs to handle the parallelism explicitly. Fortunately, highly efficient massively parallel direct solvers exist, and an implementation based on such solver may avoid dealing with parallel issues completely.

When there are only corner constraints, i.e. $D^{c}$ is empty, the BDDC preconditioner (25) - 27) reduces to

$$
M_{B D D C}: r \mapsto(I-P) E R^{c}\left(A^{c}\right)^{-1} R^{c \mathrm{~T}} E^{T}(I-P)^{\mathrm{T}} r .
$$

All coupling between substructures in the matrix $A^{c}$ is concentrated at corner degrees of freedom, while most computational work rests inside the subdomains, and an efficient solver should be able to perform it independently in parallel. Our implementation is based on the multi-frontal solver MUMPS [1, and numerical results show that this solver can indeed handle matrices of this type reasonably well. Our MATLAB implementation also uses global matrices. In both implementations, expressions involving sparse matrices are evaluated using vectors in the space $\widetilde{W^{c}}$ just as in the formulas here.

However, if there are any constraints in the globs, one has to solve the constrained system (26), and MUMPS cannot do this directly. Thus, we will transform (26) to a symmetric, positive definite system which can be solved by MUMPS. 
One way to solve system (26) is to introduce the orthogonal projection $\Pi$ onto the nullspace of $D^{c}$, which is given by

$$
\Pi=I-D^{c \mathrm{~T}}\left(D^{c} D^{c \mathrm{~T}}\right)^{-1} D^{c}
$$

As opposed to the localised analogue $\Pi_{i j}$ used in the previous section, $\Pi: \widetilde{W}^{c} \rightarrow \widetilde{W}$ is a global operator. Due to the block structure of $D^{c}$, where each block corresponds to a different glob, and because each degree of freedom belongs to at most one glob by definition, the construction of $\Pi$ can be performed in parallel.

Using projection $\Pi$, the saddle point problem 26 is equivalent to

$$
\Pi A^{c} \Pi w_{c}=\Pi R^{c \mathrm{~T}} E^{\mathrm{T}}(I-P)^{\mathrm{T}} r, \quad w_{c} \in \text { null } D^{c} .
$$

However, the operator $\Pi A^{c} \Pi$ is singular for nontrivial $D^{c}$, so we solve instead a modified system

$$
\left[\Pi A^{c} \Pi+t(I-\Pi)\right] w_{c}=\Pi R^{c \mathrm{~T}} E^{\mathrm{T}}(I-P)^{\mathrm{T}} r,
$$

where $t>0$ is a stabilization parameter, e.g. chosen as the maximal diagonal entry in $A^{c}$. Now, the operator $\Pi A^{c} \Pi+t(I-\Pi)$ is regular, while the solutions of the systems (26) and (49) are the same.

The projection $\Pi$ enforces constraints that couple all degrees of freedom on corresponding globs. For this reason, the action of $\Pi$ introduces new off-diagonal elements (called fill-in) in the projected matrix $\Pi A^{c} \Pi+t(I-\Pi)$. This is illustrated in Figure 3, where new dense off-diagonal blocks between globs appear. Because of these blocks, the performance of sparse direct solvers would seriously deteriorate. A sufficient remedy for this issue is proposed in the next section.

\section{Generalized change of variables}

To reduce the fill-in corresponding to the enforcing of the constraints following (47)-(48), we revisit and generalize the change of variables proposed in [11, 20]. On each substructure $i$, consider first the change of variables by the transformation

$$
w_{i}^{\text {new }}=\bar{H}_{i} w_{i}, \quad \bar{H}_{i}=\left[\begin{array}{cc}
\bar{U} & \bar{V} \\
0 & I
\end{array}\right] .
$$

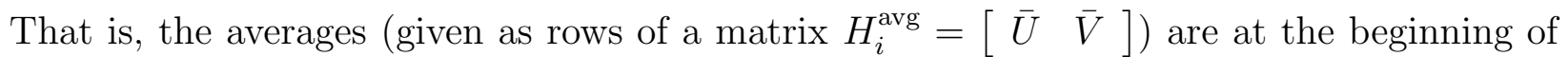
the vector $w_{i}^{\text {new }}$, replacing the variables in $w_{i}$. The remaining variables in $w_{i}$ are unchanged. We assume that the vectors of weights in the averages are linearly independent, that is, $H_{i}^{\text {avg }}$ has a full row rank. While this assumption guarantees that there exists a square submatrix of $H_{i}^{\text {avg }}$ consisting of linearly independent columns, this does not necessarily need to be the matrix $\bar{U}$, and so the inverse transformation $\bar{H}_{i}^{-1}$ may not exist. To correct this, we compute the $Q R$ decomposition of $H_{i}^{\text {avg }}$ with column pivoting to choose which variables in $w_{i}$ will be replaced by the averages. Decompose

$$
H_{i}^{\text {avg }}=Q\left[\begin{array}{ll}
U & V
\end{array}\right] K
$$


where $Q$ is an orthogonal matrix, $U$ is an upper triangular matrix, and $K$ is a permutation matrix. We now define the generalized change of variables by

$$
w_{i}^{\text {new }}=H_{i} w_{i}, \quad H_{i}=\left[\begin{array}{cc}
U & V \\
0 & I
\end{array}\right] K .
$$

Now $H_{i}^{-1}$ exists, and the inverse change of variables is defined as

$$
T_{i}=H_{i}^{-1}=K^{-1}\left[\begin{array}{cc}
U^{-1} & -U^{-1} V \\
0 & I
\end{array}\right] .
$$

The matrix $U$, though invertible, is not guaranteed to be well conditioned. This is a wellknown problem in $Q R$ decomposition [17]. However, we can drop the rows of $[U V]$ where the diagonal entry of $U$ is small, and one can argue that the constraints that were transformed into rows with negligible leading entry are (numerically) redundant. Our implementation of the change of variables uses $Q R$ decomposition by the LAPACK routine DGEQP3.

To compare with the change of variables from [11, 20], consider the case where there is just one average with unit weights. Then

$$
H_{i}^{\text {avg }}=[1, \ldots, 1], \quad U=[1], \quad K=I, \quad H_{i}=\left[\begin{array}{cccc}
1 & 1 & \ldots & 1 \\
& 1 & & \\
& & \ddots & \\
& & & 1
\end{array}\right]
$$

and we have the change of variables

$$
w_{i}=T_{i} w_{i}^{\text {new }}, \quad T_{i}=H_{i}^{-1}=\left[\begin{array}{cccc}
1 & -1 & \ldots & -1 \\
& 1 & & \\
& & \ddots & \\
& & & 1
\end{array}\right]
$$

while the transformation of variables by [11, 20] is defined as

$$
w_{i}=\left[\begin{array}{cccc}
1 & -1 & \ldots & -1 \\
1 & 1 & & \\
1 & & \ddots & \\
1 & & & 1
\end{array}\right] w_{i}^{\text {new }}
$$

With the change of basis, the BDDC preconditioner can be written as

$M_{B D D C}: r \longmapsto u=(I-P) E T R \bar{w}, \quad \bar{w} \in \widetilde{W}: \quad\langle A T \bar{w}, T z\rangle=\langle r,(I-P) E T z\rangle, \quad \forall z \in \widetilde{W}$, where $T=\operatorname{diag}\left[T_{i}\right]$. Thus, $A$ is replaced by the transformed matrix $T^{\mathrm{T}} A T$, and, by assembly at corners following (20), $A^{c}$ becomes $R^{c \mathrm{~T}} T^{\mathrm{T}} A T R^{c}$. Then, Lemma 1 yields the matrix form of the algorithm: solving the system

$$
\begin{array}{ccc}
R^{c \mathrm{~T}} T^{\mathrm{T}} A T R^{c} \bar{w}_{c}+\bar{D}^{c T} \lambda & =R^{c \mathrm{~T}} T^{\mathrm{T}} E^{\mathrm{T}} r \\
\bar{D}^{c} \bar{w}_{c} & = & 0
\end{array}
$$


followed by computation of the approximate solution $u \in \widehat{W}$ by $u=(I-P) E T R^{c} \bar{w}_{c}$. Here, the matrix $\bar{D}^{c}=D^{c} T$ is much sparser than $D^{c}$ thanks to the change of variables. It couples only the new explicit degrees of freedom on each subdomain and thus has only one +1 and one -1 entry on each row. In fact, the construction of $\bar{D}^{c}$ is similar to the construction of the operator $B$ used in FETI methods. In computations, $\bar{D}^{c}$ can be constructed directly without using either $D^{c}$ or $T$, knowing only which pairs of the (explicit) interface degrees of freedom should be coupled after the change of basis.

Instead of solving the saddle point problem (57) directly, we now use the projection as in (49) with $D^{c}$ replaced by $\bar{D}^{c}$, resulting in a new projection $\bar{\Pi}$. The sparsity structure of $\bar{\Pi}$ and of the projected matrix $\bar{\Pi} R^{c \mathrm{~T}} T^{\mathrm{T}} A T R^{c} \bar{\Pi}+\bar{t}(I-\bar{\Pi})$ are illustrated Figure 3. As can be observed, change of basis preceding the projection can lead to much lower fill-in in the off-diagonal blocks of the projected matrix.

The BDDC preconditioner can be finally rewritten in the algebraic form, which is actually used in our implementations, as follows.

Algorithm 2. The action of the BDDC preconditioner $M_{B D D C}: r \mapsto w$ with the generalized change of variables consists of solving the system

$$
\widetilde{A} \bar{w}_{c}=\bar{\Pi} R^{c \mathrm{~T}} T^{\mathrm{T}} E^{\mathrm{T}} r
$$

where

$$
\widetilde{A}=\bar{\Pi} R^{c \mathrm{~T}} T^{\mathrm{T}} A T R^{c} \bar{\Pi}+t(I-\bar{\Pi})
$$

with an arbitrary $t>0$, followed by $w=(I-P) E T R^{c} \bar{w}_{c}$.

Remark 3. Since the transformation of variables changes averages into separate degrees of freedom, one can treat these degrees of freedom as corners and assemble them just as in [11, 13, 20] to make all constraints primal. This gives no additional fill-in beyond the one caused by the change of variables, i.e., replacing $A^{c}$ by $R^{c \mathrm{~T}} T^{\mathrm{T}} A T R^{c}$. In the adaptive method (Section 3), the corners are already set and used to compute the constraints to be added adaptively. Treating all constraints as corners then requires redefining which variables are corners. This is not supported in the code described here. See Section 6 for more details.

\section{Implementation}

We have implemented the proposed method in MATLAB. Later, we have developed also a parallel version using Fortran 90 programming language and MPI.

First, we have implemented the BDDC preconditioner based on the formulation (26). In the case of corner constraints only, i.e. $D^{c}$ is empty, the method is reduced to solving a problem with matrix $A^{c}$ in each iteration. In the current version, we rely on the parallel direct solver MUMPS [1] (version 4.8.4) for this purpose.

In the implementation, two separate instances of MUMPS are necessary - one for solving problems with matrix $A^{c}$ and another for a realization of the operator $I-P$ of the discrete harmonic extension in (13) globally. The latter is equivalent to solving an independent discrete Dirichlet problem on each subdomain. 
In the case of a nontrivial matrix $D^{c}$, i.e. for additional constraints on edges and/or faces, explicit change of variables with projection (Section 5 ) is performed in parallel to form the distributed sparse matrix (59), which is then supplied to MUMPS. We have observed a great advantage in projecting the matrix after the change of variables compared to the direct projection on null $D^{c}$. It significantly decreases the computational time and memory consumption due to the reduced fill-in, as described in Section 5. In our experience, the amount of extra work needed for the transformation and the projection is typically only a small fraction of the time saved by the lower number of PCG iterations, compared to the case of corner constraints only.

Using the projection instead of re-assembling the matrix after the change of variables allows us to store the sparse matrix in memory only once, and use it in the preconditioner as well as in the PCG method, which is formulated to run on vectors from the space $\widetilde{W}^{c}$. For the preconditioner, new entries arising from the transformation and projection are stored in the memory behind the original matrix and the convention of repeated indices allowed by MUMPS is exploited.

Later, the adaptive selection of constraints described in Section 3 has been added to the implementation. As the parallelization of solving the generalized eigenvalue problems (41) on pairs of adjacent subdomains does not follow the scheme of the natural parallelization by subdomains, this part of the code has been written as a self-standing module that just passes the constraints to the main BDDC solver. Multiplication by $S_{i j}^{c}$ in the eigenvalue problem (41) is implemented by performing the interior correction on each of the two adjacent subdomains separately, and only the resulting vectors are assembled; thus, the matrices $S_{i j}^{c}$ and $A_{i j}^{c}$ for the two adjacent substructures are not formed explicitly.

\section{Numerical results}

We have tested the adaptive algorithm on several three-dimensional problems of linear elasticity coming from engineering practice. As a consistency check, we have also tested the method in two dimensions with essentially the same results as in [26]. The computations were done in MATLAB and by the parallel implementation described in Section 6. In MatLaB, the generalized eigenvalue problems for pairs of adjacent substructures were solved by explicit construction of the matrices and by standard methods for symmetric eigenvalue problems. We have also tested both the MATLAB and the $\mathrm{C}$ versions of the LOBPCG algorithm [15]. The averaging operator was constructed with weights proportional to the diagonal entries of the substructure matrices before elimination of interiors.

The first problem is a nozzle box of a ŠKODA steam turbine $28 \mathrm{MW}$ for the electric power plant Nováky, Slovakia, loaded by steam pressure. The body of the nozzle box was discretized using 2696 isoparametric quadratic finite elements with 40, 254 degrees of freedom and decomposed into 16 substructures with 37 corners, 19 edges, and 32 faces (see Fig. 4 ). Convergence of the algorithm with non-adaptive constraints is displayed in Table 1. Note that the corner coarse degrees of freedom were not sufficient to guarantee convergence. Where "3eigv" is added, constraints corresponding to three dominant eigenvalues are added at each face. This choice leads to the same number of constraints as using simple arithmetic averages. 
Comparing the last two rows in Table 1, we can see that constraints obtained from the adaptive algorithm work quite better than arithmetic averages. Our explanation is that such constraints might approximate better the direction of global eigenvectors corresponding to the extreme eigenvalues. Table 2 then contains results obtained using the adaptive selection of constraints. Each row corresponds to a different value of the threshold $\tau$, i.e. the target bound of the condition number indicator $\widetilde{\omega}$. All eigenvectors corresponding to eigenvalues greater or equal to $\tau$ were used to generate adaptive constraints. Comparing the results in Tables 1 2, we can see that the adaptive method leads to a redistribution of the number of constraints on different faces. For example, with $\tau=20$, the total number of constraints is still lower than by using arithmetic averages on all faces, while the number of iterations is improved by almost $25 \%$ and the condition number estimate $\kappa$ is improved by more than $50 \%$.

The second problem is a beam with a mesh refinement around a notch. It is discretized using 245, 687 tetrahedral finite elements with 143, 451 degrees of freedom, and decomposed into 8 substructures with 31 corners, 18 edges, and 19 faces (see Fig. 5). The results with non-adaptive constraints are summarized in Table 3, and results of the adaptive method are presented in Table 4. Comparing these two tables, we can see that, similarly as for the nozzle box problem, doubling the number of constraints reduces number of iterations to a half. Nevertheless, for both problems, the adaptive algorithm leads to a relatively small improvement in terms of the number of iterations and of the condition number estimate. This indicates that for these problems the simple arithmetic averages already work well enough, and there are no interfaces that would require extra work - the quality of the decomposition is uniform, as seen in Figs. 45 .

On the other hand, the power of the adaptive algorithm seems to be very beneficial for finite element discretization with bad aspect ratios of elements. An example of such problem is a bridge construction discretized by 39, 060 hexahedral finite elements with 157, 356 degrees of freedom, and distributed into 16 substructures with 250 corners, 30 edges, and 43 faces (see Fig. 6). The results are summarized in Tables 556. Comparing the last two rows in Table 5 we can see, that relatively poor convergence with arithmetic averages improves quite significantly when arithmetic averages over faces are replaced by the same number of adaptive averages. Moreover, from Table 6 we see that, e.g., doubling the number of constraints with $\tau=5$ decreases the number of iterations more than six times, and with $\tau=2$ the number of iterations is reduced more than ten times while the number of constraints increases approximately four times.

In order to test the performance of the algorithm in the presence of jumps in material coefficients, we have created a problem of a cube with material parameters $E=10^{6} \mathrm{~Pa}$ and $\nu=0.45$, penetrated by four bars with parameters $E=2.1 \cdot 10^{11} \mathrm{~Pa}$ and $\nu=0.3$, consisting of 107, 811 degrees of freedom, and distributed into 8 substructures with 30 corners, 16 edges, and 15 faces (see Fig. 7 and note that the bars cut the substructures only through faces). Similar problems are solved in practice to determine numerically (anisotropic) properties of composite materials. Comparing the results in Tables 7 and 8 we can see, that with $\tau=10,000$ only 10 additional averages over faces are used to decrease the number of 
iterations 2.6 times. With $\tau=2$, the number of iterations is decreased 10 times compared to the non-adaptive algorithm with arithmetic averages over all globs $(\mathrm{c}+\mathrm{e}+\mathrm{f})$, whereas the number of constraints is increased less than 3 times.

To test the parallel behaviour of the MUMPS solver at our applications, we run the solver only with corner coarse degrees of freedom on a benchmark problem consisting of

cubic subdomains, the number of which is growing in two dimensions (see Fig. 8). Since the size of the subdomains is fixed, the problem fixed at one side and loaded at the opposite side is changing its nature, and consequently, some growth in number of iterations is expected. The sequence of problems is run on an increasing number of processors, which matches the number of subdomains. Presented computations were performed on $1.5 \mathrm{GHz}$ Intel Itanium 2 processors of SGI Altix 4700 computer in CTU Supercomputing Centre, Prague.

We can see in Table 9, that after a jump in times between 16 and 25 subdomains related probably to the computer's architecture, the times of analysis, factorization, as well as per one iteration remain almost constant. The "total wall time" includes also the second factorization of MUMPS for computing the discrete harmonic extensions and all I/O operations.

In the presented algorithm, a considerable amount of work is spent by generating the adaptive constraints. This part, which consists of solving local eigenproblems, may eventually dominate the whole computation. Each of these eigenproblems is common to a pair of subdomains, so the parallelism is different from the "natural" subdomain-wise parallelism of domain decomposition. For this reason, an independent distribution of eigenproblems among processors is performed, and each processor which solves an eigenproblem is linked with the two processors which store the data of subdomains within the pair.

An investigation of scaling of this part of the implementation on a variable number of processors was performed for the problem of turbine nozzle box with 16 subdomains. In Fig. 9, the summary for the sequence of $2^{k}, k=0,1,2, \ldots, 5$, processors is shown.

\section{Conclusion}

The adaptive BDDC method has been presented. The paper contains several original contributions. First, the definition of space where BDDC runs is given as the nullspace of a global matrix of constraints. For an efficient and straightforward implementation of this formulation, a generalization of the change of variables is proposed. This allows an efficient handling of multiple arbitrary constraints on a substructure face. This functionality is required for the implementation of constraints that are generated adaptively. The adaptive selection of constraints from [26] has been reformulated in a mathematically equivalent way to use only the operators of BDDC and to match the overall approach of the rest of this paper to minimize programming requirements. The adaptive method is based on simultaneous solution of generalized eigenvalue problems defined for each face in the decomposition. The eigenvalues serve as a condition number indicator, so the minimal number of constraints is added to guarantee that the condition number indicator is below a given threshold. The corresponding eigenvectors are used to derive the coefficients of the constraints. Numerical experiments confirm that the eigenvalues provide a good prediction of the final condition number of the preconditioned operator. 
A parallel implementation of the method has been developed and presented. It is based on a global formulation of the matrix of the BDDC preconditioner, and it is built on top of solver MUMPS, which provides most of the parallelism and minimizes custom coding. The implementation has been tested on a number of problems of 3D elasticity. Results for several real world problems are included.

In our experiments, adaptive BDDC has shown to be quite powerful. Many times, it has been able to save the situation for poorly selected corners, even in the case of disconnected subdomains, the situation often faced in real applications of domain decomposition when using graph partitioners. Adaptive BDDC is able to handle very ill-conditioned problems (e.g. problems with jumps in coefficients, complicated geometries with deformed elements, etc.), which are almost impossible to solve by standard BDDC method using only arithmetic averages on edges and faces. Such problems would either require a prohibitive number of PCG iterations or may not converge at all. This class of problems is the target application of the proposed method, as the extra cost of generating the constraints adaptively is not negligible and would not pay for well-conditioned problems.

The solution of local eigenproblems by LOBPCG in generation of the adaptive constraints requires many iterations and accounts for most of the time for some problems. A suitable preconditioning of these eigenproblems to reduce the number of LOBPCG iterations will be studied elsewhere.

\section{Acknowledgement}

We would like to thank to Jaroslav Kruis, Jaroslav Novotný, and Jan Leština for providing us with real engineering problems. We are also grateful to Marian Brezina for visualization

of some meshes by his own package MyVis. Part of this work was done when Jakub Šístek was visiting University of Colorado Denver.

This work was supported in part by National Science Foundation under grants DMS-0713876 and CNS-0719641, by Czech Science Foundation under grant GA ČR 106/08/0403, and by Academy of Sciences of the Czech Republic under grant AV0Z10190503.

\section{References}

[1] P. R. Amestoy, I. S. Duff, J.-Y. L'Excellent, Multifrontal parallel distributed symmetric and unsymmetric solvers, Comput. Methods Appl. Mech. Engrg. 184 (2000) 501-520.

[2] S. C. Brenner, L.-Y. Sung, BDDC and FETI-DP without matrices or vectors, Comput. Methods Appl. Mech. Engrg. 196 (8) (2007) 1429-1435.

[3] M. Brezina, C. Heberton, J. Mandel, P. Vaněk, An iterative method with convergence rate chosen a priori, UCD/CCM Report 140, University of Colorado at Denver, Denver, CO, http://ccm.ucdenver.edu/reports/rep140.pdf (1999). 
[4] J.-M. Cros, A preconditioner for the Schur complement domain decomposition method, in: I. Herrera, D. E. Keyes, O. B. Widlund (eds.), Domain Decomposition Methods in Science and Engineering, National Autonomous University of Mexico (UNAM), México, 2003, pp. 373-380, 14th International Conference on Domain Decomposition Methods, Cocoyoc, Mexico, January 6-12, 2002.

[5] J. W. Demmel, Applied Numerical Linear Algebra, Society for Industrial and Applied Mathematics (SIAM), Philadelphia, PA, 1997.

[6] C. R. Dohrmann, A preconditioner for substructuring based on constrained energy minimization, SIAM J. Sci. Comput. 25 (1) (2003) 246-258.

[7] C. Farhat, M. Lesoinne, K. Pierson, A scalable dual-primal domain decomposition method, Numer. Linear Algebra Appl. 7 (2000) 687-714.

[8] C. Farhat, J. Mandel, The two-level FETI method for static and dynamic plate problems. I. An optimal iterative solver for biharmonic systems, Comput. Methods Appl. Mech. Engrg. 155 (1-2) (1998) 129-151.

[9] J. Fish, V. Belsky, Generalized aggregation multilevel solver, Internat. J. Numer. Methods Engrg. 40 (23) (1997) 4341-4361.

[10] Y. Fragakis, M. Papadrakakis, The mosaic of high performance domain decomposition methods for structural mechanics: Formulation, interrelation and numerical efficiency of primal and dual methods, Comput. Methods Appl. Mech. Engrg. 192 (2003) 3799-3830.

[11] A. Klawonn, O. Rheinbach, A parallel implementation of dual-primal FETI methods for three dimensional linear elasticity using a transformation of basis, SIAM J. Sci. Comput. 28 (5) (2006) 1886-1906.

[12] A. Klawonn, O. Rheinbach, O. B. Widlund, An analysis of a FETI-DP algorithm on irregular subdomains in the plane, SIAM J. Numer. Anal. 46 (5) (2008) 2484-2504.

[13] A. Klawonn, O. B. Widlund, Dual-primal FETI methods for linear elasticity, Comm. Pure Appl. Math. 59 (11) (2006) 1523-1572.

[14] A. Klawonn, O. B. Widlund, M. Dryja, Dual-primal FETI methods for three-dimensional elliptic problems with heterogeneous coefficients, SIAM J. Numer. Anal. 40 (1) (2002) 159-179.

[15] A. V. Knyazev, Toward the optimal preconditioned eigensolver: locally optimal block preconditioned conjugate gradient method, SIAM J. Sci. Comput. 23 (2) (2001) 517-541, copper Mountain Conference (2000).

[16] J. Kruis, Domain decomposition methods for distributed computing, Saxe-Coburg Publications, Kippen, Stirling, Scotland, 2006. 
[17] J. Langou, Private communication (2008).

[18] P. Le Tallec, J. Mandel, M. Vidrascu, Balancing domain decomposition for plates, Contemp. Math. 180 (1994) 515-524, proceedings of the 7th International Symposium on Domain Decomposition Methods, Penn State, November 1993.

[19] P. Le Tallec, J. Mandel, M. Vidrascu, A Neumann-Neumann domain decomposition algorithm for solving plate and shell problems, SIAM J. Numer. Anal. 35 (1998) 836-867.

[20] J. Li, O. B. Widlund, FETI-DP, BDDC, and block Cholesky methods, Internat. J. Numer. Methods Engrg. 66 (2) (2006) 250-271.

[21] J. Mandel, Intelligent block iterative methods, in: J. Robinson (ed.), FEM Today and the Future, Robinson and Associates, 1993, pp. 471-477, proceedings of the 7th World Congress on Finite Elements, Monte Carlo, November 1993.

[22] J. Mandel, An iterative solver for $p$-version finite elements in three dimensions, Comput. Methods Appl. Mech. Engrg. 116 (1-4) (1994) 175-183, iCOSAHOM'92 (Montpellier, 1992).

[23] J. Mandel, C. R. Dohrmann, Convergence of a balancing domain decomposition by constraints and energy minimization, Numer. Linear Algebra Appl. 10 (7) (2003) 639-659.

[24] J. Mandel, C. R. Dohrmann, R. Tezaur, An algebraic theory for primal and dual substructuring methods by constraints, Appl. Numer. Math. 54 (2) (2005) 167-193.

[25] J. Mandel, B. Sousedík, Adaptive coarse space selection in the BDDC and the FETI-DP iterative substructuring methods: Optimal face degrees of freedom, in: O. B. Widlund, D. E. Keyes (eds.), Domain Decomposition Methods in Science and Engineering XVI, Lecture Notes in Computational Science and Engineering, vol. 55, Springer-Verlag, 2006, pp. 421-428.

[26] J. Mandel, B. Sousedík, Adaptive selection of face coarse degrees of freedom in the BDDC and the FETI-DP iterative substructuring methods, Comput. Methods Appl. Mech. Engrg. 196 (8) (2007) 1389-1399.

[27] J. Mandel, B. Sousedík, BDDC and FETI-DP under minimalist assumptions, Computing 81 (2007) 269-280.

[28] J. Mandel, B. Sousedík, C. R. Dohrmann, Multispace and multilevel BDDC, Computing 83 (2-3) (2008) 55-85.

[29] J. Mandel, R. Tezaur, On the convergence of a dual-primal substructuring method, Numer. Math. 88 (2001) 543-558. 
[30] J. Mandel, R. Tezaur, C. Farhat, A scalable substructuring method by Lagrange multipliers for plate bending problems, SIAM J. Numer. Anal. 36 (5) (1999) 1370-1391.

[31] C. Pechstein, R. Scheichl, Analysis of FETI methods for multiscale PDEs, Numer. Math. 111 (2) (2008) 293-333.

[32] C. Pechstein, R. Scheichl, Analysis of FETI methods for multiscale PDEs - Part II: Interface variations, Numerische Mathematik, submitted (2009).

[33] G. Poole, Y.-C. Liu, J. Mandel, Advancing analysis capabilities in ANSYS through solver technology, Electronic Transactions on Numerical Analysis 15 (2003) 106-121.

[34] J. Šístek, J. Novotný, J. Mandel, M. Čertíková, Burda, P., BDDC by a frontal solver and stress computation in a hip joint replacement, Math. Comput. Simulation 80 (6) (2010) 1310-1323.

[35] B. F. Smith, P. E. Bjørstad, W. D. Gropp, Domain decomposition: parallel multilevel methods for elliptic partial differential equations, Cambridge University Press, Cambridge, 1996.

[36] B. Sousedík, Comparison of some domain decomposition methods, Ph.D. thesis, Czech Technical University in Prague, Faculty of Civil Engineering, Department of Mathematics (2008).

[37] B. Sousedík, J. Mandel, On the equivalence of primal and dual substructuring preconditioners, Electron. Trans. Numer. Anal. 31 (2008) 384-402. URL http://etna.mcs .kent.edu/vol.31.2008/pp384-402.dir/pp384-402.html

[38] A. Toselli, O. B. Widlund, Domain Decomposition Methods-Algorithms and Theory, vol. 34 of Springer Series in Computational Mathematics, Springer-Verlag, Berlin, 2005.

[39] O. B. Widlund, Accomodating irregular subdomains in domain decomposition theory, in: M. Bercovier, M. Gander, R. Kornhuber, O. Widlund (eds.), Domain Decomposition Methods in Science and Engineering XVIII, vol. 70 of Lecture Notes in Computational Science and Engineering, Springer-Verlag, 2009, proceedings of 18th International Conference on Domain Decomposition, Jerusalem, Israel, January 2008. 


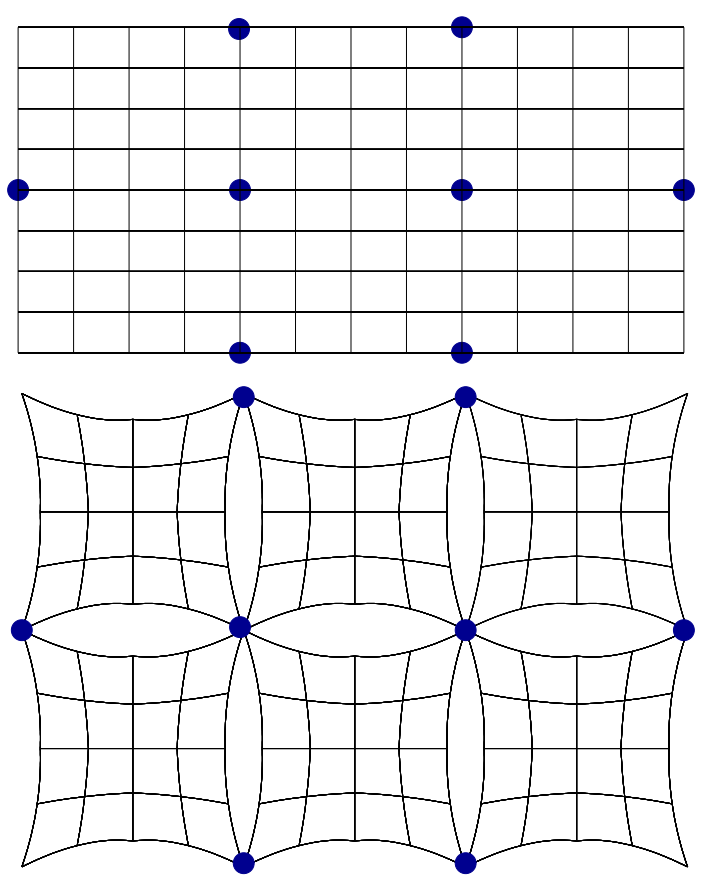

Figure 1: Example of an actual mesh (top) and the corresponding fictitious mesh for construction of space $W^{c}$ (bottom), the dots mark corners.

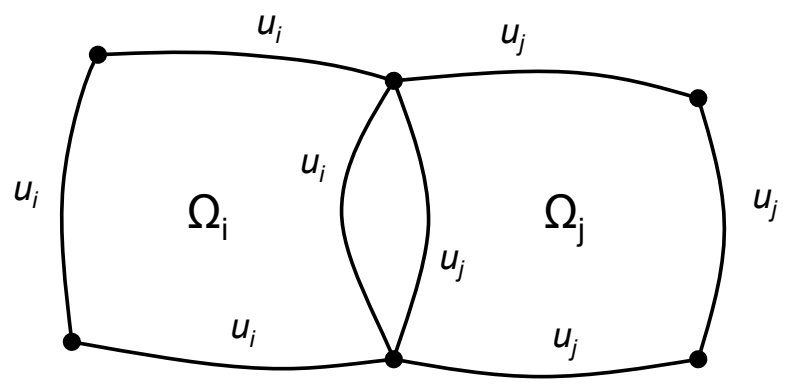

Figure 2: Illustration of two adjacent subdomains in 2D for the computation of the condition number indicator. 


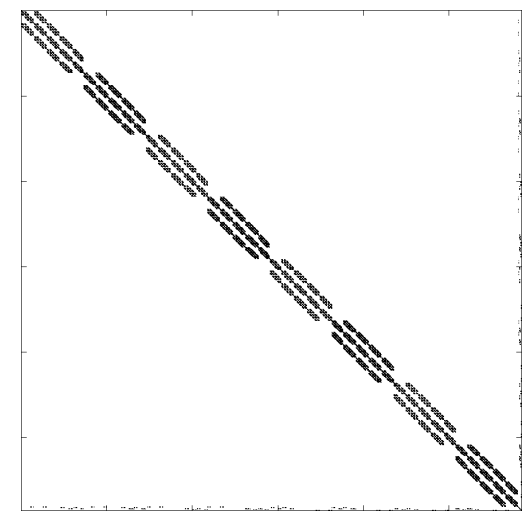

(a) $\mathrm{nnz}=136,937$

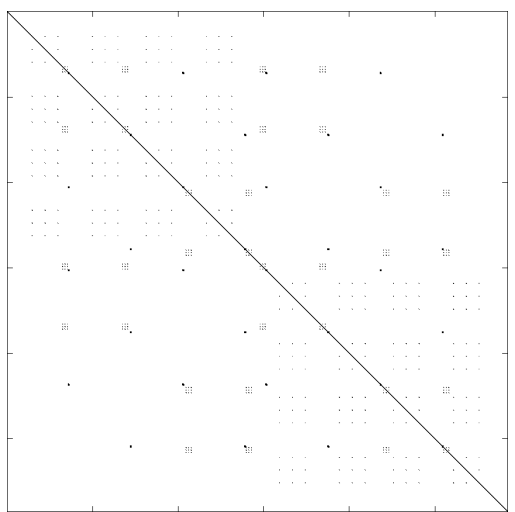

(c) $\mathrm{nnz}=5301$

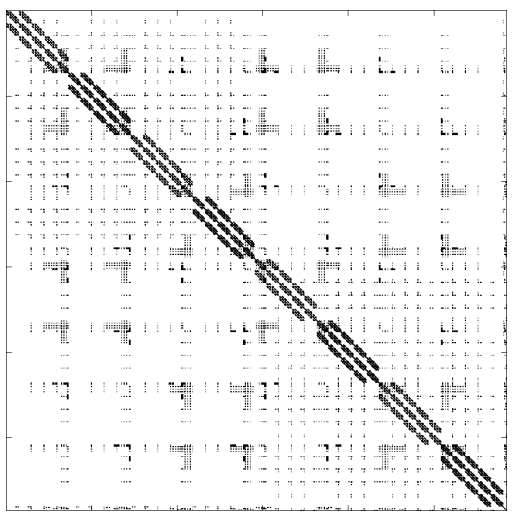

(e) $\mathrm{nnz}=228,954$

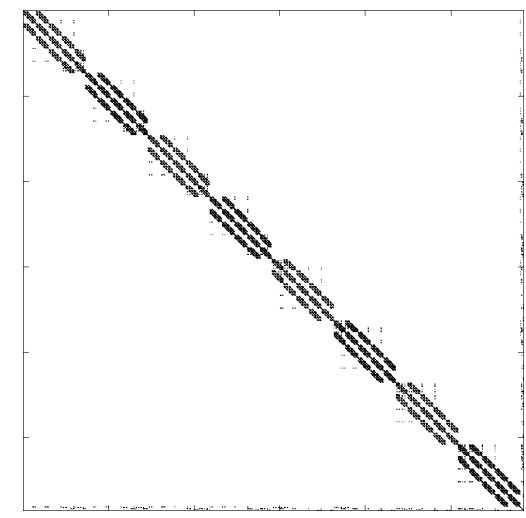

(b) $\mathrm{nnz}=141,773$

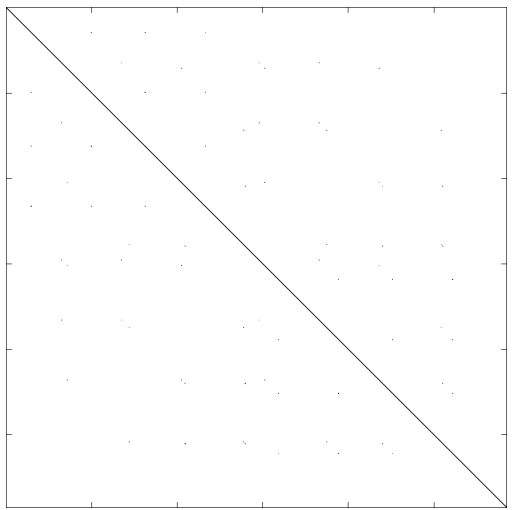

(d) $\mathrm{nnz}=3141$

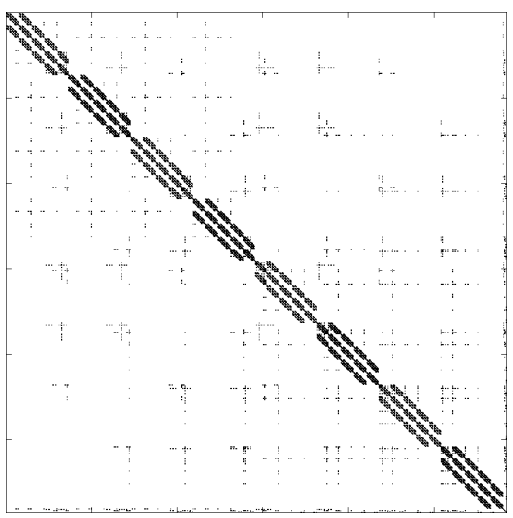

(f) $\mathrm{nnz}=156,982$

Figure 3: Sparsity patters for 3D elasticity problem for a cube decomposed into $2 \times 2 \times 2$ substructures $(H / h=4)$ with 7 corners, 6 edges, 12 faces, and 2187 degrees of freedom. The matrix $D^{c}$ (resp. $\bar{D}^{c}$ ) contains 54 rows to enforce the equality of arithmetic averages over edges. The matrices (a), (c), (e) are in the original degrees of freedom, while (b), (d), (f) are after the change of variables $(53)$ : the operators $A^{c}$ in panel (a) and $R^{c \mathrm{~T}} T^{\mathrm{T}} A T R^{c}$ in panel (b), projections $\Pi$ in panel (c) and $\bar{\Pi}$ in panel (d), and projected operators $\Pi A^{c} \Pi+t(I-\Pi)$ in panel (e) and $\bar{\Pi} R^{c \mathrm{~T}} T^{\mathrm{T}} A T R^{c} \bar{\Pi}+\bar{t}(I-\bar{\Pi})$ in panel (f). All are square matrices with size 2925 . 

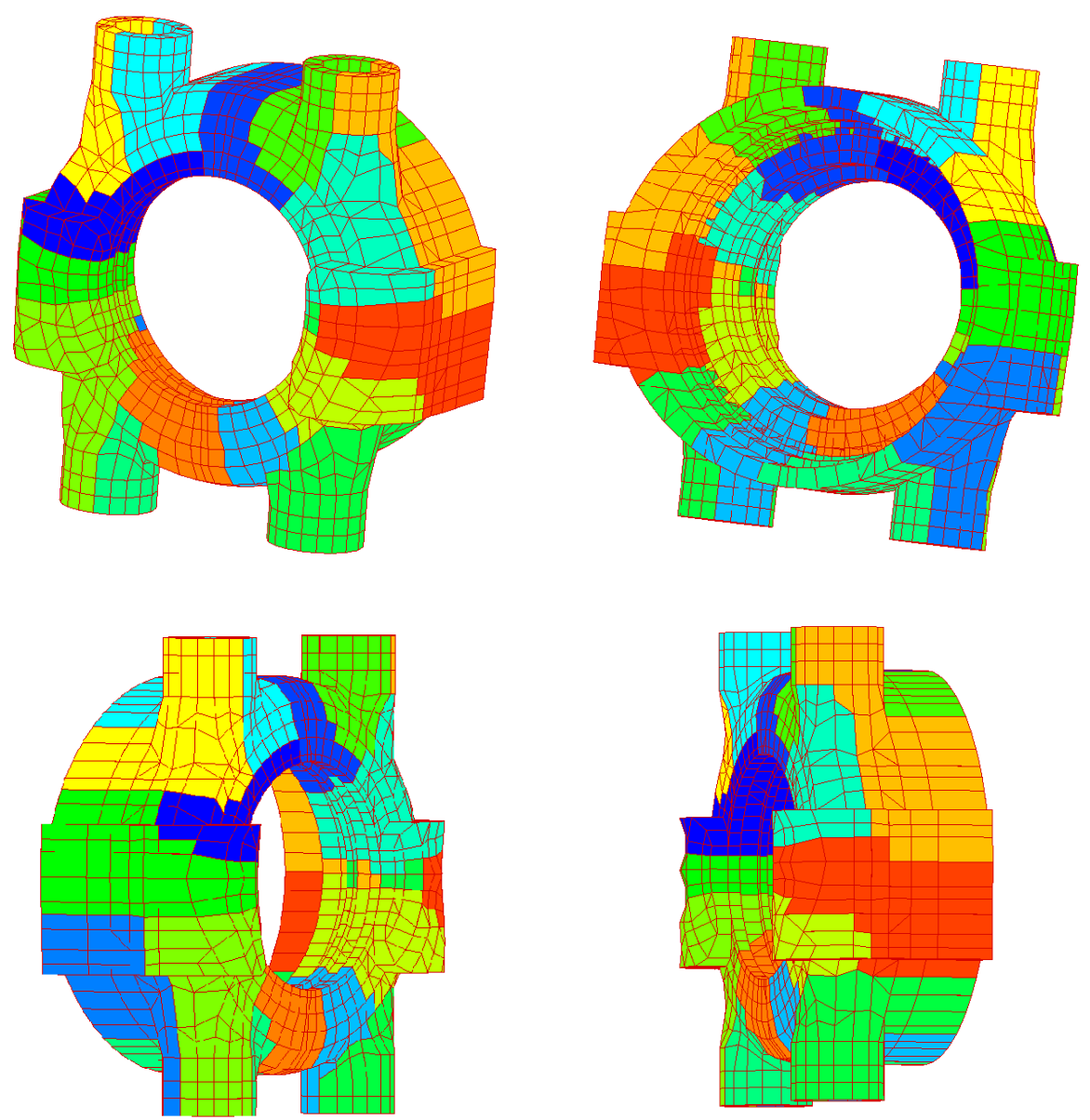

Figure 4: Finite element discretization and substructuring of the nozzle box, consisting of 40, 254 degrees of freedom, 16 substructures, 37 corners, 19 edges, and 32 faces.

\begin{tabular}{c|r|r|r} 
constraints & $N c$ & $\kappa$ & $i t$ \\
\hline $\mathrm{c}$ & 0 & $\mathrm{NA}$ & $\mathrm{NA}$ \\
$\mathrm{c}+\mathrm{e}$ & 117 & 1021.7 & 103 \\
$\mathrm{c}+\mathrm{e}+\mathrm{f}$ & 213 & 40.3 & 47 \\
$\mathrm{c}+\mathrm{e}+\mathrm{f}$ (3eigv) & 213 & 26.5 & 40
\end{tabular}

Table 1: Results for the turbine nozzle box problem. The first three rows correspond to non-adaptive approach with corner constraints and arithmetic averages over edges/faces, and the last row corresponds to corner constraints with arithmetic averages over edges and three weighted averages over faces obtained from eigenvectors of the local generalized eigenvalue problems, $N c$ is number of constraints (rows in the matrix $D$ ), $\kappa$ is the approximate condition number estimate from the Lanczos sequence in conjugate gradients, and $i t$ is the number of iterations for relative residual tolerance $10^{-8}$. 


\begin{tabular}{r|r|r|r|r}
$\tau$ & $\widetilde{\omega}$ & $N c$ & $\kappa$ & $i t$ \\
\hline$\infty(=\mathrm{c}+\mathrm{e})$ & $\mathrm{NA}$ & 117 & 1021.690 & 103 \\
50 & 49.772 & 158 & 44.8781 & 48 \\
20 & 19.824 & 200 & 16.8938 & 36 \\
10 & 9.965 & 274 & 11.171 & 27 \\
5 & 4.998 & 408 & 8.820 & 20
\end{tabular}

Table 2: Results for the turbine nozzle box problem using the adaptive approach. $\tau$ is the threshold, and $\widetilde{\omega}$ is the condition number indicator from (38). The other headings are same as in Table 1.
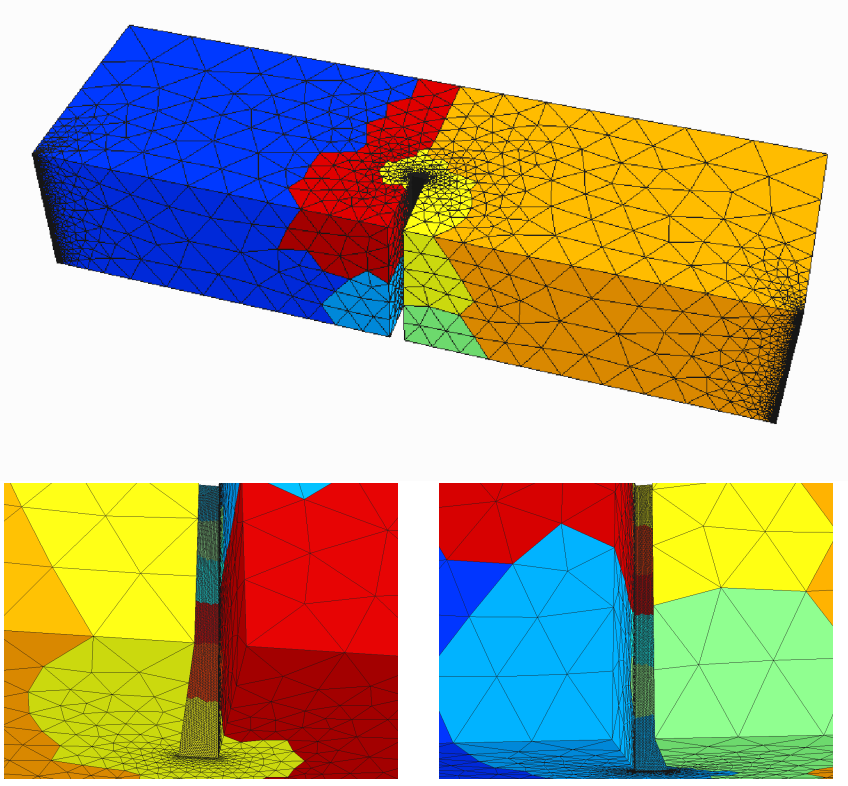

Figure 5: Finite element discretization and substructuring of the beam with a notch, consisting of 143, 451 degrees of freedom, 8 substructures, 31 corners, 18 edges, and 19 faces.

\begin{tabular}{c|r|r|r} 
constraint & $N c$ & $\kappa$ & $i t$ \\
\hline $\mathrm{c}$ & 0 & 127.1 & 79 \\
$\mathrm{c}+\mathrm{e}$ & 111 & 101.0 & 61 \\
$\mathrm{c}+\mathrm{e}+\mathrm{f}$ & 168 & 22.4 & 32 \\
$\mathrm{c}+\mathrm{e}+\mathrm{f}($ 3eigv $)$ & 168 & 13.2 & 30
\end{tabular}

Table 3: Results for the beam with a notch. The headings are same as in Table 1 . 


\begin{tabular}{r|r|r|r|r}
$\tau$ & $\widetilde{\omega}$ & $N c$ & $\kappa$ & $i t$ \\
\hline$\infty(=\mathrm{c}+\mathrm{e})$ & 149.0 & 111 & 101.0 & 61 \\
20 & 18.272 & 119 & 19.011 & 41 \\
10 & 9.986 & 134 & 8.505 & 31 \\
5 & 4.994 & 163 & 4.655 & 24 \\
3 & 2.998 & 215 & 2.873 & 18 \\
2 & 1.993 & 340 & 2.145 & 14
\end{tabular}

Table 4: Results for the beam with a notch. The headings are same as in Table 2.
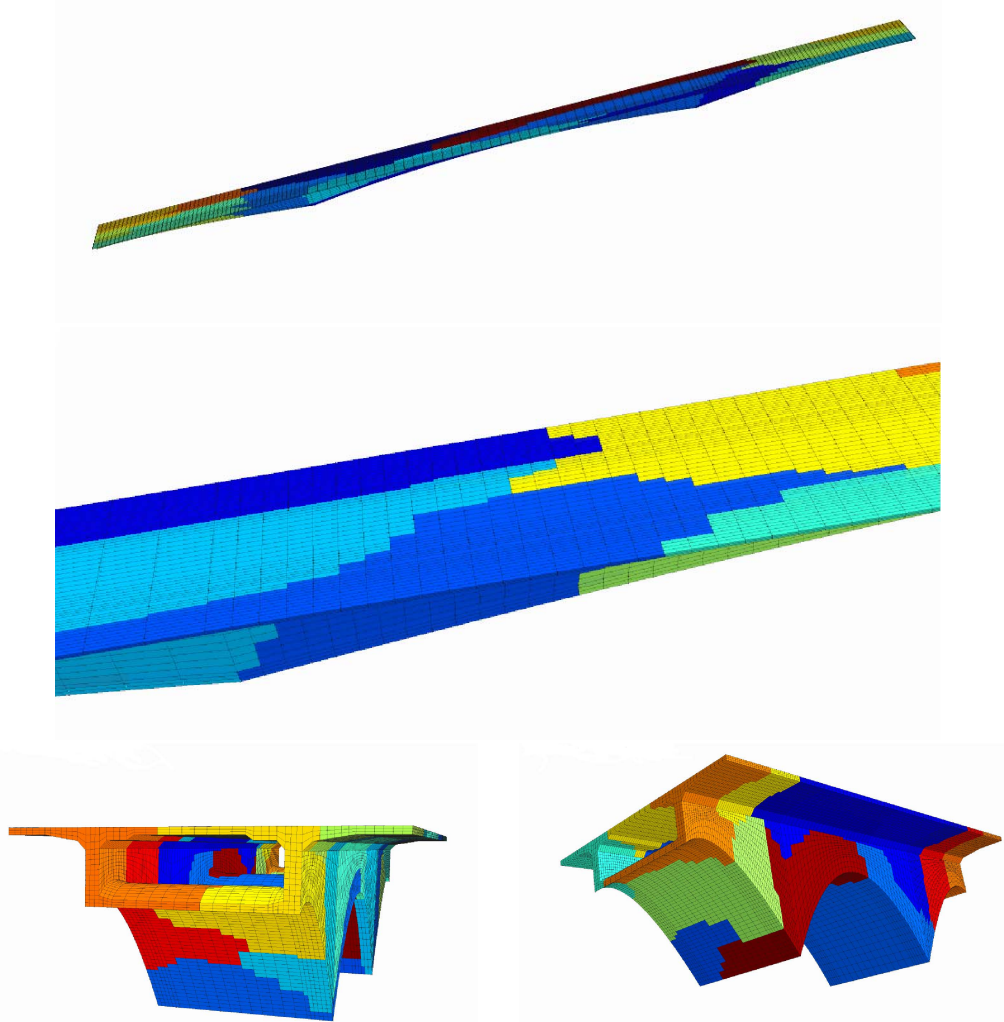

Figure 6: Finite element discretization and substructuring of the bridge construction, consisting of 157, 356 degrees of freedom, 16 substructures, 250 corners, 30 edges, and 43 faces.

\begin{tabular}{c|r|r|r} 
constraint & $N c$ & $\kappa$ & $i t$ \\
\hline $\mathrm{c}$ & 0 & 2301.4 & 224 \\
$\mathrm{c}+\mathrm{e}$ & 180 & 2252.4 & 220 \\
$\mathrm{c}+\mathrm{e}+\mathrm{f}$ & 309 & 653.6 & 160 \\
$\mathrm{c}+\mathrm{e}+\mathrm{f}(3 e i g v)$ & 309 & 177.8 & 103
\end{tabular}

Table 5: Results for the bridge construction. The headings are same as in Table1. 


\begin{tabular}{r|r|r|r|r}
$\tau$ & $\widetilde{\omega}$ & $N c$ & $\kappa$ & $i t$ \\
\hline$\infty(=\mathrm{c}+\mathrm{e})$ & 6500.5 & 180 & 2252.4 & 220 \\
650 & 589.338 & 185 & 483.517 & 169 \\
30 & 29.568 & 292 & 28.739 & 64 \\
5 & 4.997 & 655 & 5.014 & 26 \\
2 & 1.998 & 1301 & 2.011 & 14
\end{tabular}

Table 6: Results for the bridge construction. The headings are same as in Table 2 .
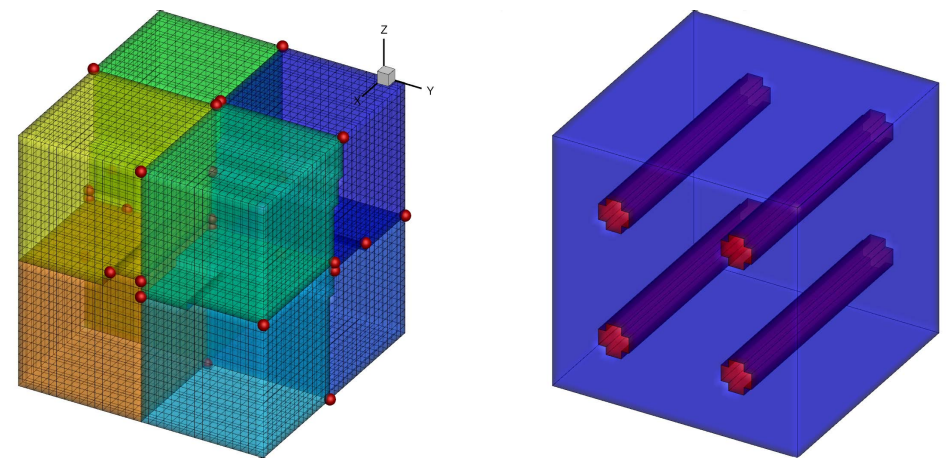

Figure 7: Finite element discretization and substructuring of the cube with jumps in coefficients, consisting of 107,811 degrees of freedom, 8 substructures, 30 corners, 16 edges, and 15 faces.

\begin{tabular}{c|r|r|r} 
constraint & $N c$ & $\kappa$ & $i t$ \\
\hline $\mathrm{c}$ & 0 & $408,101.0$ & 326 \\
$\mathrm{c}+\mathrm{e}$ & 108 & $125,390.0$ & 234 \\
$\mathrm{c}+\mathrm{e}+\mathrm{f}$ & 153 & $18,914.9$ & 169 \\
$\mathrm{c}+\mathrm{e}+\mathrm{f}(3$ eigv $)$ & 153 & 1266.4 & 71
\end{tabular}

Table 7: Results for the cube with jumps in coefficients. The headings are same as in Table 1 .

\begin{tabular}{r|r|r|r|r}
$\tau$ & $\widetilde{\omega}$ & $N c$ & $\kappa$ & $i t$ \\
\hline$\infty(=\mathrm{c}+\mathrm{e})$ & $270,000.0$ & 108 & $125,390.0$ & 234 \\
10,000 & 5145.293 & 118 & 1843.35 & 90 \\
1,000 & 380.019 & 129 & 173.562 & 35 \\
100 & 77.189 & 132 & 6.423 & 24 \\
5 & 4.990 & 173 & 4.362 & 20 \\
2 & 1.998 & 451 & 2.803 & 16
\end{tabular}

Table 8: Results for the cube with jumps in coefficients. The headings are same as in Table 2 


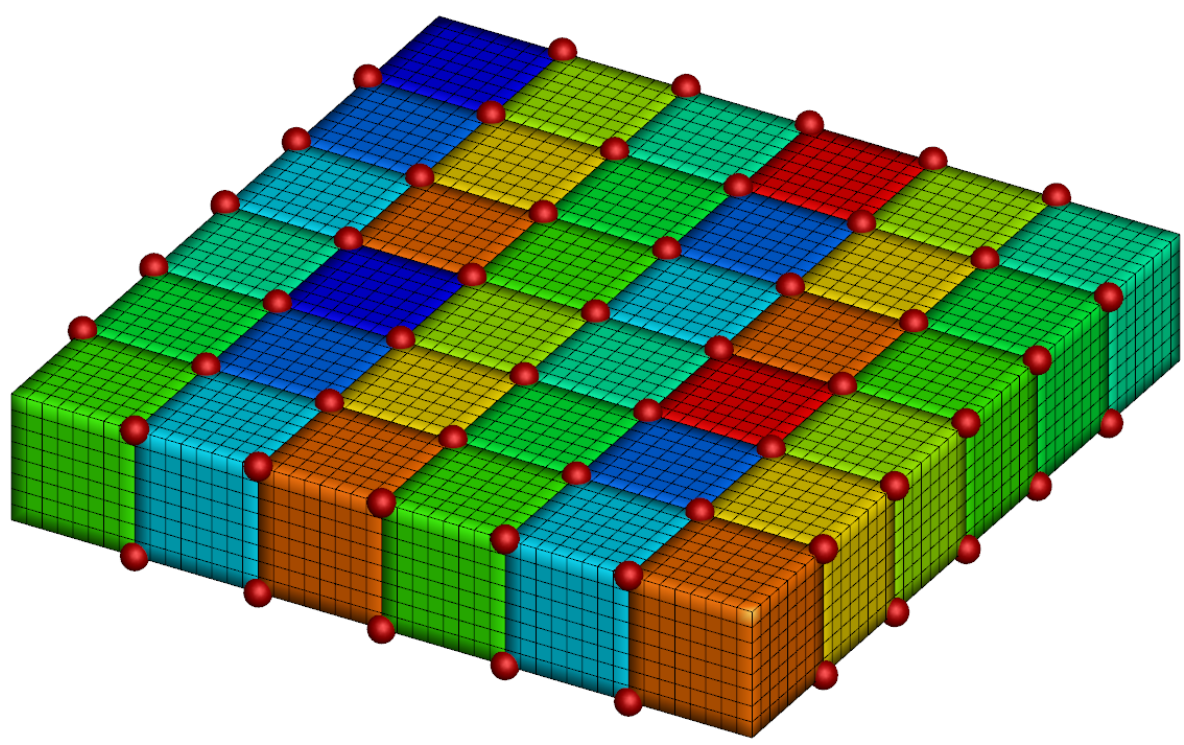

Figure 8: Example of a configuration of planar cubes test problem with 36 subdomains and $H / h=8$, red dots represent corners.

\begin{tabular}{c|c|c|c|c|c|c|c} 
number of subdomains & 4 & 9 & 16 & 25 & 36 & 49 & 64 \\
degrees of freedom & 7803 & 16,875 & 29,403 & 45,387 & 64,827 & 87,723 & 114,075 \\
\hline condition number est. & 28.3 & 38.0 & 42.2 & 44.4 & 45.7 & 46.5 & 47.1 \\
number of PCG iterations & 13 & 26 & 36 & 42 & 44 & 46 & 47 \\
\hline analysis by MUMPS (sec) & 0.2 & 0.5 & 1 & 15 & 14 & 16 & 19 \\
factorization by MUMPS (sec) & 0.5 & 0.4 & 0.8 & 12 & 10 & 12 & 14 \\
PCG iterations (sec) & 0.8 & 3.6 & 13 & 613 & 524 & 579 & 643 \\
\hline one PCG iteration (sec) & 0.06 & 0.14 & 0.4 & 15 & 12 & 13 & 14 \\
\hline total wall time (sec) & 3 & 6 & 19 & 715 & 616 & 696 & 794
\end{tabular}

Table 9: Weak scaling on planar cubes problem (e.g. Fig. 8), corners only, $H / h=8$. 


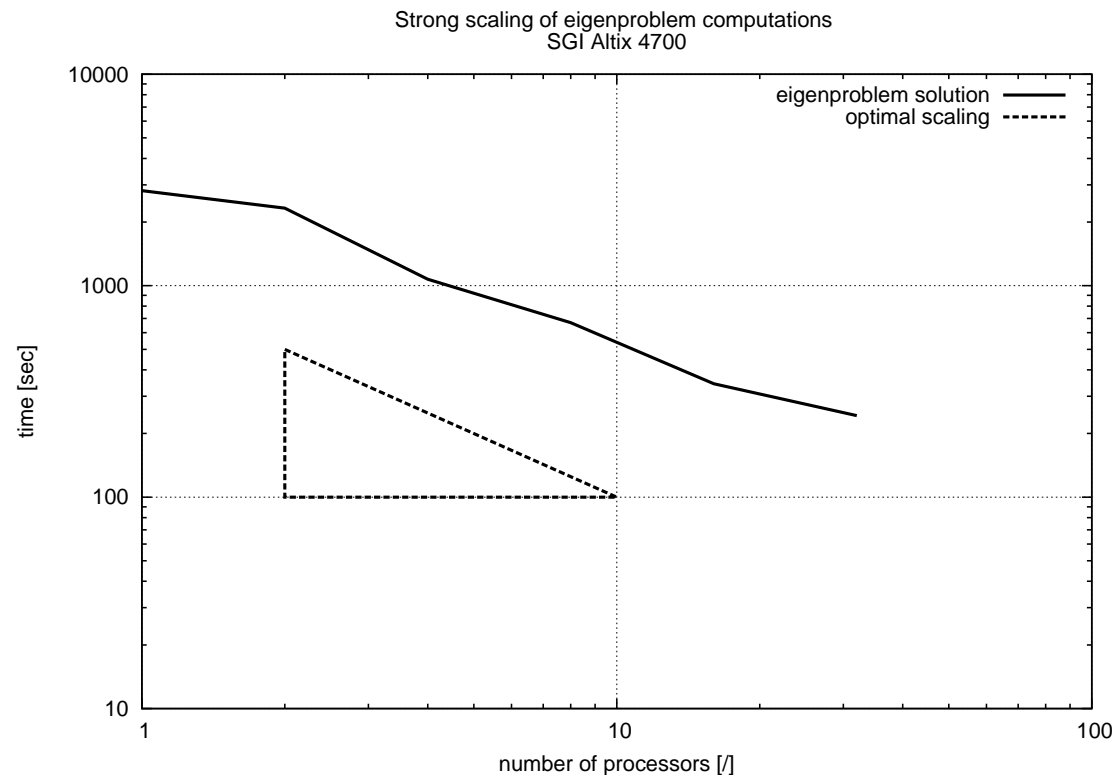

Figure 9: Dependence of the computational time on the number of processors for solution of local eigenproblems, nozzle box problem, 16 subdomains, 30 eigenproblems. 